\title{
Full-disk magnetic oscillations in the solar photosphere
}

\author{
P. F. Moretti ${ }^{1}$, A. Cacciani ${ }^{2}$, A. Hanslmeier ${ }^{3}$, M. Messerotti ${ }^{4}$, W. Otruba ${ }^{5}$, and A. Warmuth ${ }^{3}$ \\ 1 INAF, Osservatorio Astronomico di Capodimonte, via Moiarello 16, 80131 Napoli, Italy \\ 2 Department of Physics University of Rome, P.le A. Moro 2, 00185 Rome, Italy \\ ${ }^{3}$ Institute of Geophysics, Astrophysics and Meteorology of the University of Graz, Universitaetsplatz 5, 8010 Graz, Austria \\ ${ }^{4}$ INAF, Osservatorio Astronomico di Trieste, via G. B. Tiepolo 11, 34131 Trieste, Italy \\ 5 Kanzelhöhe Solar Observatory, 9521 Treffen, Austria
}

Received 4 October 2002 / Accepted 27 February 2003

\begin{abstract}
Photospheric observations of the magnetic oscillations have been carried out to research magneto-acoustic waves in spots. The coupling between the magnetic field and the $p$-modes has been studied using reduced areas on the solar disk, but a global scenario is still missing. Four hour full-disk magnetograms (at 4 arcsec/pix spatial resolution) obtained in the sodium D-lines were analysed pixel-by-pixel (locally) and using the spherical harmonics decomposition (globally).

Magnetic oscillations were detected at different frequencies and identified on the solar disk. The global properties of the magnetic oscillations, shown via the $\ell-v$ diagrams, are for the first time presented and discussed.
\end{abstract}

Key words. Sun: oscillations — Sun: magnetic fields

\section{Introduction}

For centuries the solar magnetic field has been thought to be almost stationary, when the 22 year cycle was considered the only relevant change. Since a few decades, a more complete understanding of the processes that lead to the solar cycle changes and the heating of the corona has emerged through the observation of the dynamic small scale magnetic structures of the Sun. Nevertheless, the measurement of small magnetic fluxes and their interpretation in terms of the magnetic field is not a trivial task. Most of our knowledge of the fast evolving magnetic structures has been obtained by indirect observations in the radio or in the Fraunhofer lines formed in the chromosphere, where the magnetic energy dominates.

In the photosphere, the observed magnetic field fluctuations have been observed during the research of magneto-acoustic waves in spots (Lites 1986; Lites 1992; Lites et al. 1998; Horn et al. 1997; Cacciani et al. 1998; Ruedi et al. 1998; Norton \& Ulrich 2000; Zhugzhda et al. 2000; Norton et al. 2001), and in the quiet sun (Ulrich 1996; Hasan et al. 2000; Bellot Rubio et al. 2001; Norton et al. 2001). Much observational evidence seems to suggest inhibition of the velocity fluctuations by the magnetic activity: local seismology in magnetic regions has shown absorption of the $p$-modes power at low frequencies and its enhancement at the higher ones (Lites et al. 1982; Braun \& Lindsey 1999; Thomas \& Stanchfield 2000). Detailed works have shown the coupling between the velocity oscillations and

Send offprint requests to: P. F. Moretti, e-mail: moretti@na.astro.it the magnetic field (Bogdan et al. 1996; Jain \& Haber 2002 and references therein), but the global properties have not yet been shown. Only the solar mean magnetic field as recorded in integrated sunlight has been presented (Garcia et al. 1999; Scherrer et al. 1977).

In this paper we present the characteristics of the full-disk magnetic $(B)$ and velocity $(V)$ oscillations detected in the NaI D lines at $4.3 \mathrm{arcsec} / \mathrm{pix}$ spatial resolution. The data have been analysed pixel-by-pixel and through the spherical harmonic decomposition to obtain the power and $B-V$ phase difference $l-v$ diagrams. A preliminary interpretation of the power of the magnetic field oscillations is presented.

\section{The data and the $B-V$ crosstalk}

At the Kanzelhöhe Solar Observatory, simultaneous full-disk Dopplergrams, and intensity and longitudinal magnetic field images were obtained by a system based on a sodium MagnetoOptical Filter (MOF). The MOF provides two narrow passbands (35 mA FWHM each) on the opposite wings of the solar NaI D1 and D2 profiles at a distance of approximately $70 \mathrm{m \AA}$ from the central resonance wavelength, named hereafter the blue (Blue) and the red (Red) passbands. The Blue and Red are alternatively transmitted by an electronic modulator. Another modulator in front of the telescope selects the opposite circular polarized solar sigma profiles $\left(\sigma^{+}\right.$and $\left.\sigma^{-}\right)$and four images are successively acquired at video rate: the $B l u e^{+}, \operatorname{Red}^{+}$, $B l u e^{-}, \mathrm{Red}^{-}$. These 4 primitive images are processed to provide in $160 \mathrm{~ms}$ an intensity image as $B l u e^{+}+\operatorname{Red}^{+}+\mathrm{Blue}^{-}+\mathrm{Red}^{-}$, a Dopplergram as Blue ${ }^{+}-\operatorname{Red}^{+}+B l u e^{-}-\operatorname{Red}^{-}$, and a longitudinal 
magnetic field image as $B l u e^{+}-\operatorname{Red}^{+}-B l u e^{-}+\operatorname{Red}^{-}$. Images are averaged to reach a high $S / N$ and typically $40 \mathrm{~s}$ integrated images are needed for a 16 bit Dopplergram (for details, see Cacciani et al. 1999 and references therein).

The spatial resolution is $4.3 \mathrm{arcsec} / \mathrm{pix}$.

The data we show consist of two sets of simultaneous dopplergrams and longitudinal magnetograms obtained on January 30th, 1998. Different days were analysed and the results are similar for all the days in that period of the solar cycle. The images were acquired every minute and $256 \mathrm{~min}$ were selected for the analysis. All the images were registered; the differential rotation was not removed and the maximum sweep at disk center corresponds to five pixels.

Dopplergrams were calibrated as described in Moretti and the MOF Development Group (2000). Magnetograms were calibrated as Dopplergrams, using the Zeeman displacement between the sigma components as a simple Doppler shift. A second-order calibration is applied to take into account the broadening of the solar profile in the presence of a magnetic field (Marmolino et al. 1997; Cacciani \& Moretti 1997).

Even when this second-step calibration is used, a crosstalk between the magnetic and velocty signals is present. This $B-$ $V$ crosstalk arises from the fact that only two passbands (the Blue and Red) are sampled along the solar profile at different wavelengths depending on the relative offset velocity between the solar surface and the observer.

A detailed discussion about the effects of the crosstalk between the intensity $(I)$ and $V$ has been presented in Moretti \& Severino (2002).

To first order, any signal can be written as a linear combination of the measured quantity and of other factors. For example, $B=\beta_{(B, v \text { off })} B_{0}+\alpha_{(B, v o f f)} V_{0}+\theta_{(B, v \text { off })} I_{0}$. The coefficients mainly depend on the instrumental characteristics and on the wavelength tuned by the relative offset velocity $v_{\text {off }}$. The computation of the coefficients relative to the true quantity is usually required for the first-step calibration of the signal, while the other two are properly related to the crosstalk.

In this context, we do not want to correct the images for the induced spurious signals (as shown in Moretti \& Severino 2002), but our intent is to estimate the error due to the crosstalk terms, especially in the $B-V$ phase difference, in order to interpret correctly the results of the data analysis.

The velocity signal can be written as $S_{v}=c_{v} V_{0}+\frac{\delta V_{0}}{\delta B} \Delta B$, where $V_{0}$ is the true velocity, $c_{v}$ the sensitivity of the instrument computed taking into account the true passbands of the filters and the solar line profile.

If the solar line changes its profile due to magnetic broadening (Marmolino et al. 1995; Ulrich et al. 1993; Cacciani \& Moretti 1997), the sensitivity $c_{v}$ changes mainly due to the changes of the slope of the line. As a result, the induced velocity signal due to the magnetic influence can be written as $\Delta V=\left(c_{v}{ }^{0}-c_{v}{ }^{B}\right) V_{0}=a \frac{\delta c_{v}}{\delta B} \Delta B$, where $c_{v}{ }^{0}$ and $c_{v}{ }^{B}$ are the Dopplergram sensitivities for the quiet and magnetic lines: respectively.

The estimate of the velocity signal $\Delta V$ induced by a magnetic fluctuation depends on the model adopted to generate the modified solar profiles in the presence of $B$. We used the model presented in Cacciani \& Moretti (1997), developed for

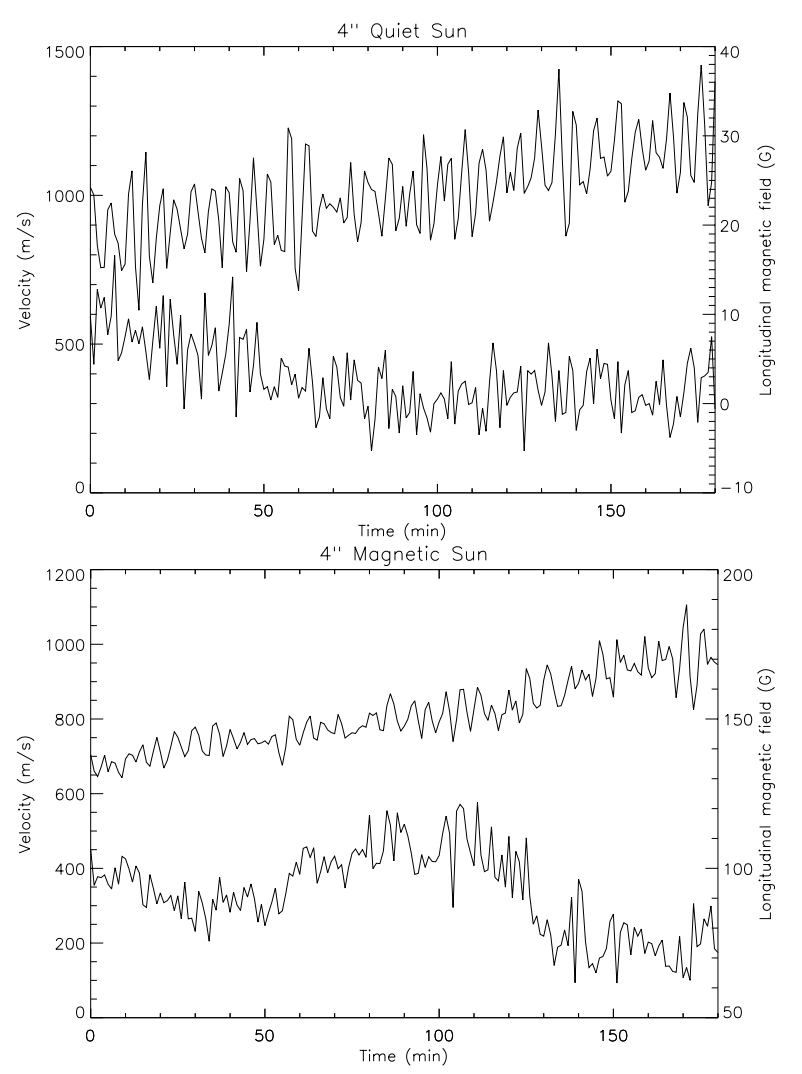

Fig. 1. One-minute cadence velocity (top curves) and magnetic (bottom curves) time-series for a $4^{\prime \prime} \times 4^{\prime \prime}$ quiet (top) and magnetic (bottom) regions.

the sodium $\mathrm{D}$ lines. We obtain $\Delta V=0.4 \Delta B \frac{\mathrm{m} \mathrm{s}^{-1}}{G}$, (for velocities between -2000 and $2000 \mathrm{~m} \mathrm{~s}^{-1}$ ).

Analogously, the induced magnetic signal due to a velocity shift of the tuned wavelength is $\Delta B=b \frac{\delta c_{v}}{\delta v} \Delta V$. The $a$ and $b$ scaling factors are those used to calibrate the images.

In Figs. 1 and 2 typical time-series and the computed magnetic signal induced by a $100 \mathrm{~m} \mathrm{~s}^{-1}$ velocity Doppler shift as a function of the velocity offset are shown. In our case, a $100 \mathrm{~m} \mathrm{~s}^{-1}$ amplitude oscillation induces a maximum $0.05 \mathrm{G}$ spurious fluctuation of the measured magnetic field, or in general $\Delta B=5 \times 10^{-4} \Delta V \frac{G}{\mathrm{~m} \mathrm{~s}^{-1}}$.

\section{The analysis and the results}

The full-disk data have been treated locally (that is, pixel-by-pixel, maintaining the spatial resolution, Lites et al. 1999) and globally (using the spherical harmonics decomposition) to obtain the spatial distribution of the powers and of the $B-V$ phase difference, and the $\ell-v$ diagrams. The daily trend was removed in the pixel-by-pixel time-series using a polynomial fit, while a differential filter was used in the spherical harmonics decomposition. The final power and phase spectra were corrected for these filters of the time-series.

Let us consider what a $\ell-v$ diagram is: the velocity images (or other signals) are filtered by masks and the coefficients time-series of this decomposition are fast Fourier transformed (FFT). The spectra as a function of the frequency and of the spherical degree are then displayed. In helioseismology, 


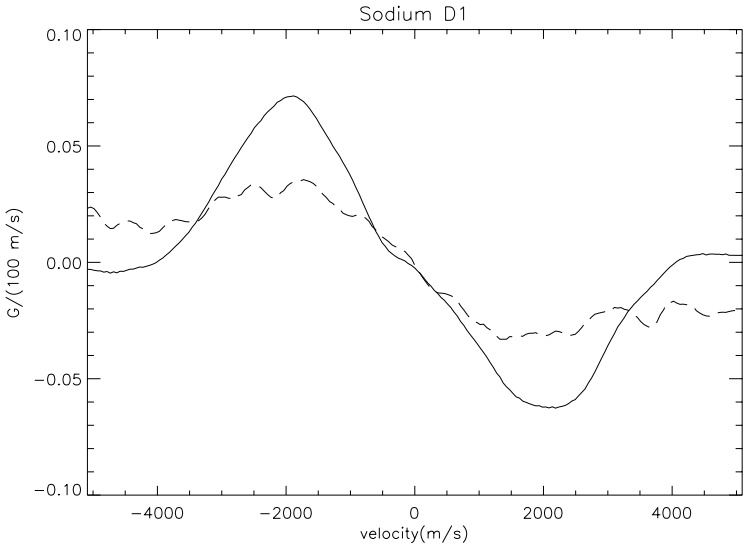

Fig. 2. The magnetic signal induced by a velocity oscillation of $100 \mathrm{~m} \mathrm{~s}^{-1}$ shifting the reference wavelength tuned by the velocity offset (on $x$-axis). The plot is shown for a sodium MOF at $270^{\circ}$ on the D1 for the Dopplergrams obtained as $\log ($ Blue/Red) (solid) or as (Blue-Red)/(Blue+Red) (dashed).

the velocity and intensity coefficients are interpreted as the global resonance of the pressure and temperature fluctuations.

When the oscillations are treated locally, they are different as the mixing with the local phenomena and their characteristic distributions on the disk are not eliminated by a filter as the spherical harmonics.

The local analysis uses a three-dimensional representation, as the distribution on the disk (in $x, y$ ) is shown as function of the frequency $(v)$. The image time-series are pixel-by-pixel fast Fourier transformed (FFT) and the power and phase difference maps are obtained at each frequency. This kind of analysis permits us to investigate the distribution on the disk of the oscillating power and phase but cannot intrinsically achieve a high frequency resolution. In fact, long time-series would produce a spatial average because of the solar rotation and of the evolution of the structures on the solar surface. The granules and supergranules have time-scales of $10 \mathrm{~min}$ and hours with dimensions of $1^{\prime \prime}$ and tenths of arcseconds respectively. The structures would lose their identity after a lifetime and a compromise has to be reached so as not to remove anything but the rotation. As a consequence, we selected approximately four hour long observation runs and the frequency resolution is limited to $65 \mu \mathrm{Hz}$.

\subsection{The pixel-by-pixel analysis}

We first discuss the results from the pixel-by-pixel analysis. The powers and $B-V$ phase difference maps (computed as described in Sect. 4) were obtained.

Some examples are shown in Fig. 4 for one of the two selected areas shown in Fig. 3. The phase difference changes along the frequency domain and depends strongly on the spatial resolution. Any estimate, averaged in frequency or space, could give misleading values (see Fig. 5 and Fig. 1 in Moretti et al. 2001). Results can depend on the techniques used by the different researches for the determination of the phase values, as described in Norton et al. (2001).

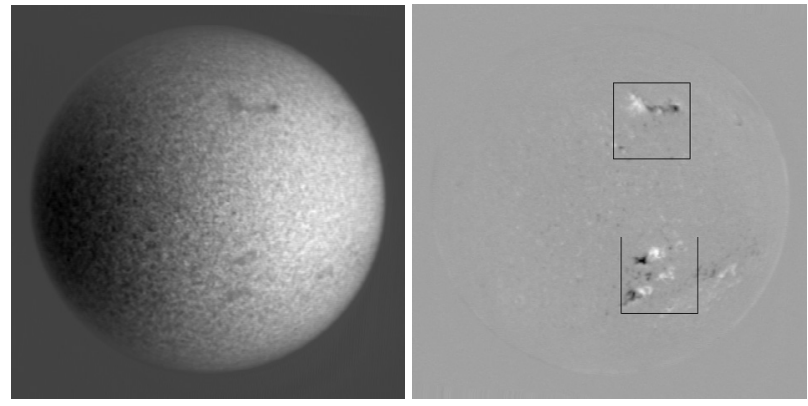

Fig.3. A Dopplergram (left) and a magnetogram (right) obtained on 30th January 1998 at Kanzelhöhe. The area in the upper frame in the magnetogram is shown in Fig. 4.

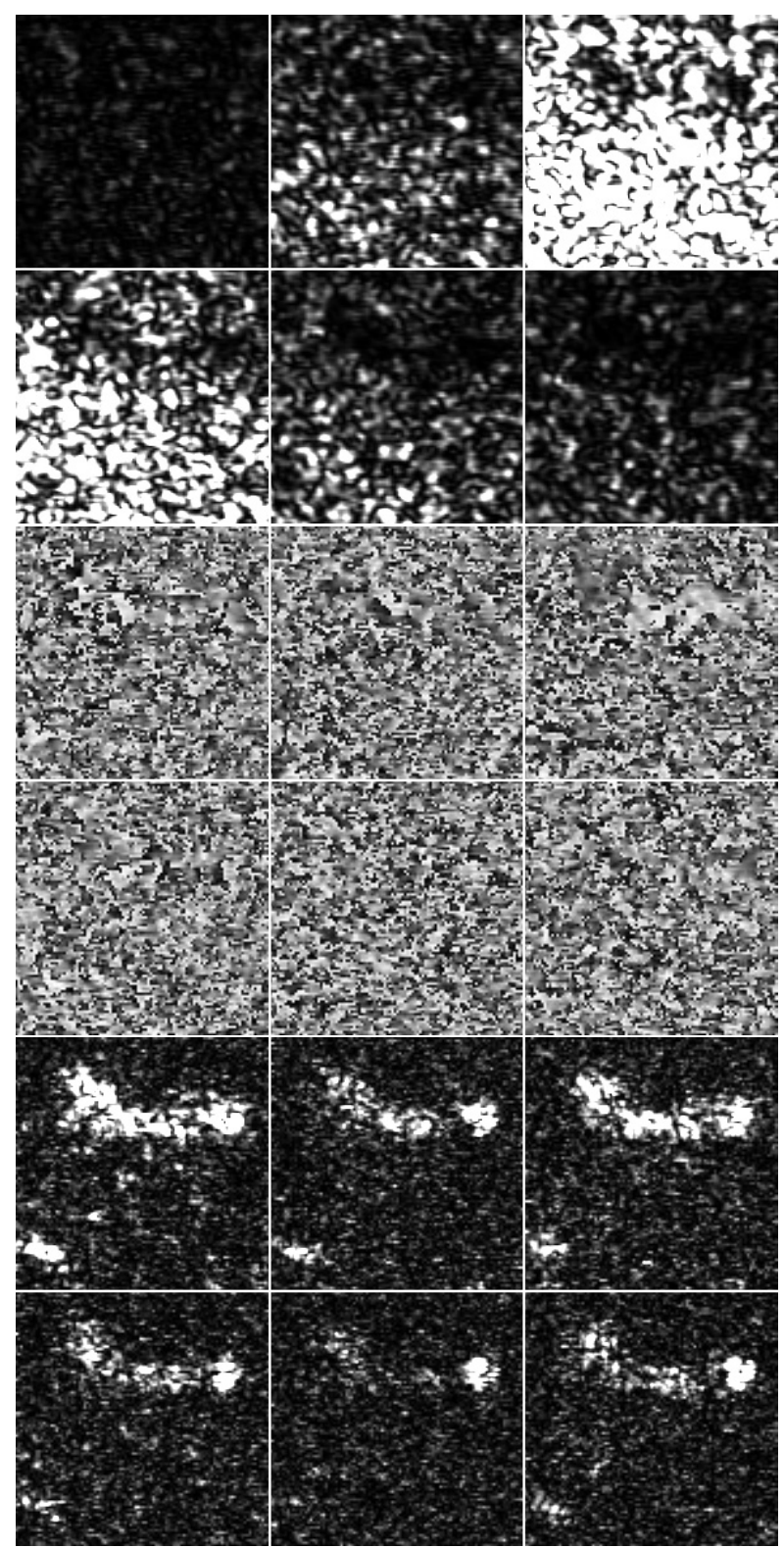

Fig. 4. From the pixel-by-pixel analysis: the velocity power (top), the $B-V$ phase difference (center) and the longitudinal magnetic power (bottom) for the selected upper area in Fig. 3 at 0.91, 2.47, $3.16,3.78,4.88,5.53 \mathrm{mHz}$ (from top left to bottom right). The black to white scales are: for the velocity power from 0 to $200\left(\mathrm{~m} \mathrm{~s}^{-1}\right)^{2}$, for the phase from -180 to +180 degrees, for the magnetic power from 0 to $0.4 \mathrm{G}^{2}$. 


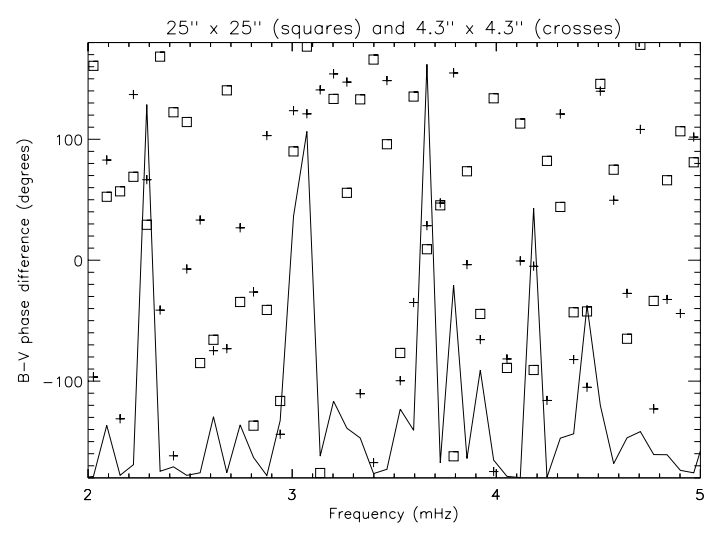

Fig. 5. The $B-V$ phases for a quiet region $(B \simeq 10 \mathrm{G})$ and the product between the velocity and magnetic power is superimposed (solid). The squares refer to an integration of the time-series over a $25^{\prime \prime} \times 25^{\prime \prime}$ area and the crosses to its central pixel $\left(4.3^{\prime \prime} \times 4.3^{\prime \prime}\right)$. When many peaks at different frequencies are present and when the magnetic elements are not resolved, the measured phases can vary widely (see the values at $\simeq 4.2 \mathrm{mHz}$ ).

When a phenomenon is not well distinguished from others (or noise) because of the limited spatial or frequency resolution, special techniques have to be applied to avoid the mixing between different contributions.

Often the coherence is used to estimate the statistical reliability of a phase value, but it strongly depends on the algorithm used to build it. In the case of the pixel-by-pixel analysis, the coherence can be chosen in different ways to estimate the statistical consistence of a phase value (from different time-series, different frequency ranges etc.). In our case, we compared the results from different days but we did not segment the 4 hour time series in order to maintain a reasonable frequency resolution to distinguish the 5 min solar oscillations peaks. The results, similar for different days, show a very fuzzy $B-V$ phase at 4 arcsec resolution. This suggests that the signals at this spatial scale are dominated by noise and not by an ensemble coherent wave motion. Analogously, the same analysis at larger scales (up to $20^{\prime \prime} \times 20^{\prime \prime}$, averaging more pixels) does not show any significant change from the noisy phase values in the quiet sun regions.

In Fig. 6 the power spectra for a plage and spot region are displayed. The 3-min power revealed in many measurements is confirmed (Schmieder et al. 2000; Lites 1986; Damé et al. 1984; Thomas et al. 1982; Beckers \& Schultz 1972).

\subsection{The $\ell-v$ diagrams}

The $\ell-v$ diagrams were obtained for data sets of $256 \mathrm{~min}$ in January 1998 and using two different software packages to decompose the images into spherical harmonics: the GRASP managed by the GONG group and the OAC package developed by M. Oliviero (see Fig. 7).

The general properties of the power are summarised as follows:

1) the magnetic power diagram does not reproduce the velocity diagram (where the $p$-mode ridges dominate) at $\ell<200$;

2) the magnetic power diagram shows two bumps at the five and three-minute bands (Fig. 9).

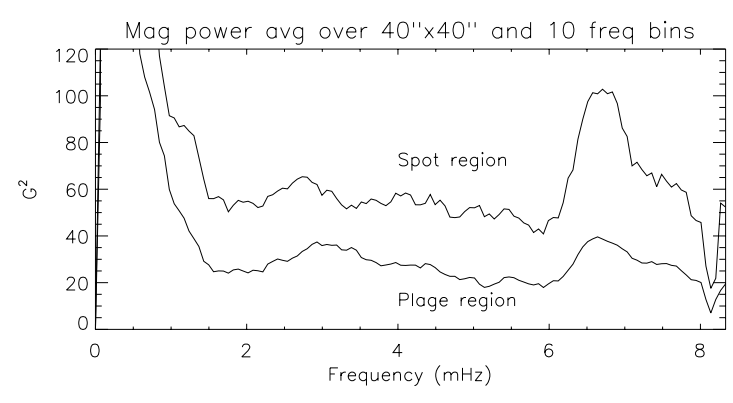

Fig. 6. The local magnetic power for a spot region and a plage region of approximately $40^{\prime \prime} \times 40^{\prime \prime}$. The five and three-minute bumps are visible.
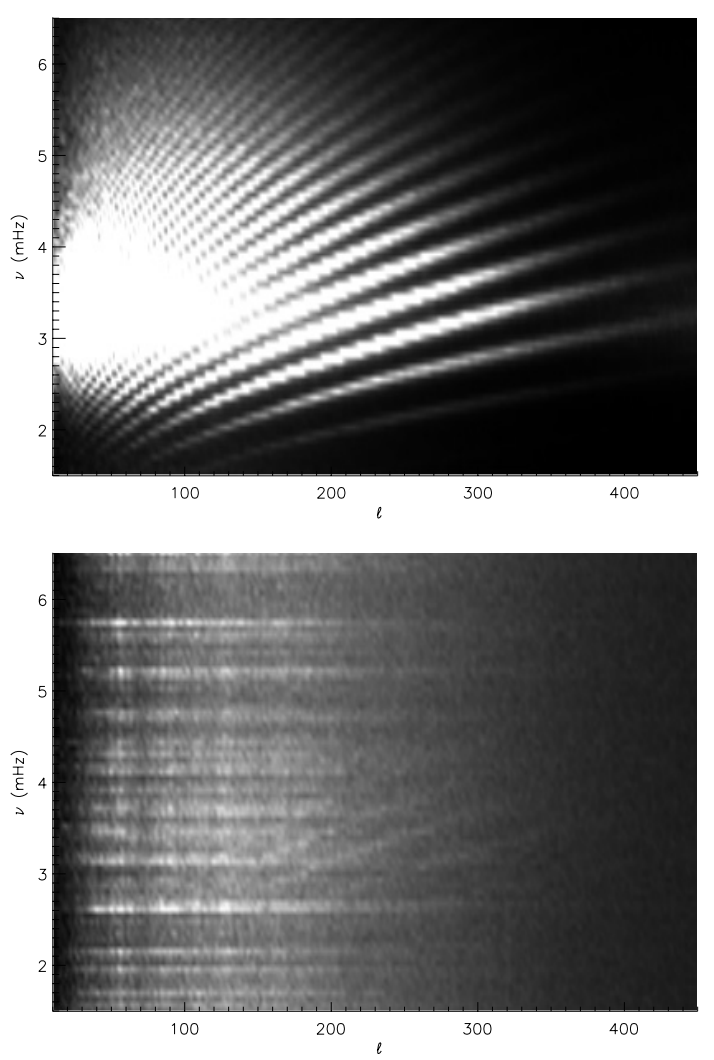

Fig. 7. The $\ell-v$ diagrams for the velocity (top) and longitudinal magnetic field power (bottom). They have been obtained from 256 images acquired each minute. The scales from black to white correspond to $\left[6 \times 10^{-4}\right]\left(\mathrm{m} \mathrm{s}^{-1}\right)^{2} / \mathrm{Hz}$ and $\left[4 \times 10^{-8}\right](\mathrm{G})^{2} / \mathrm{Hz}$ respectively. The software was provided by M. Oliviero of the OAC.

Both these properties are confirmed by the pixel-by-pixel analysis, where the magnetic spectra do not mimic the velocity power (Fig. 1) and show an increased high frequency power corresponding to the magnetic structures (see Fig. 6).

We mention that the presence of the 3-min bump in the $\ell-v$ magnetic spectra does not imply a global response of the solar cavity (likewise in the 5-min range for the $p$-modes) but can be reproduced by a broad spatial distribution of local sources on the surface (as irregular shaped plages etc.). This can be also seen in the increase of the power in correspondence to the typical spatial scales of the plages and spots on the solar disk during the observing days. 

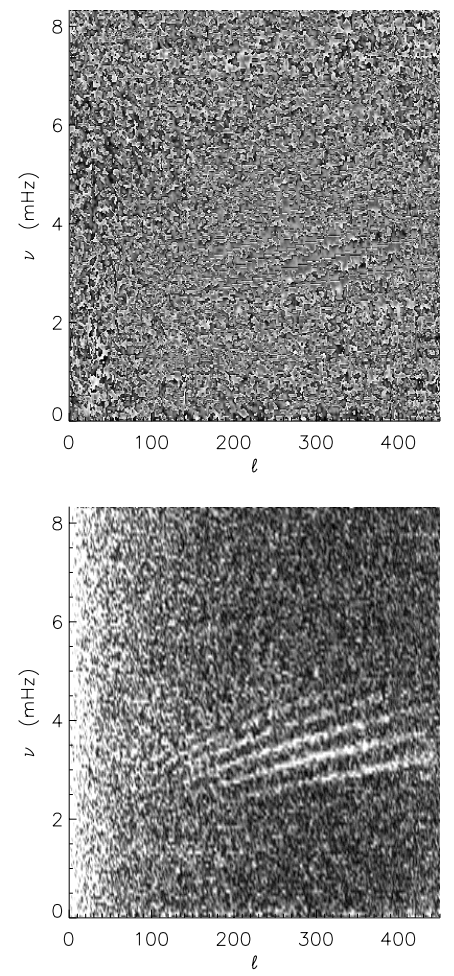

Fig. 8. The $\ell-v$ diagrams for the $B-V$ phase (top) and coherence (bottom) relative to the powers diagrams in Fig. 4. The black and white scale spans from $-180^{\circ}$ to $180^{\circ}$ and from 0.0 to 0.2 respectively.

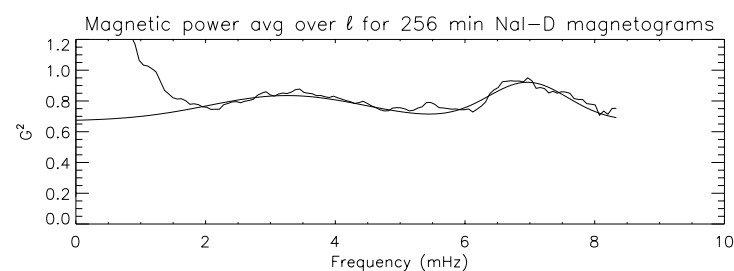

Two gaussian components

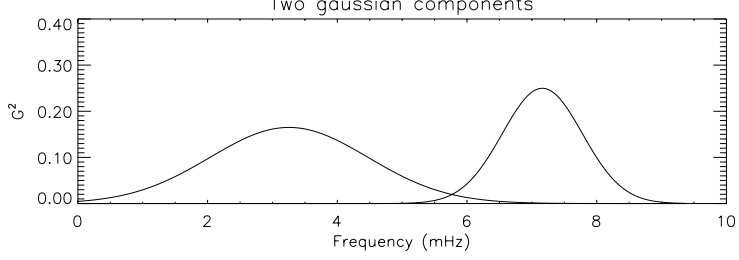

Fig. 9. Top: the $\ell-v$ diagram averaged over $\ell$.The smooth line is a fit containing the two Gaussian components shown in the bottom panel added to a bias equal to $0.67 \mathrm{G}^{2}$.

\section{The $B-V$ phase}

The phase difference between the $B$ and $V$ signals is $\phi_{b_{v}}=$ $a \tan \frac{\left(\operatorname{Im} \tilde{B}_{0} \tilde{V}_{0}^{*}\right)}{\left.\operatorname{Re} \tilde{B}_{0} \tilde{V}_{0}^{*}\right)}$, where the $\tilde{B}$ is the Fourier transformed $B$ and $\tilde{V}^{*}$ the conjugate of the Fourier transformed $V$. In the presence of the crosstalk terms, it changes to $\phi_{b_{v}}=a \tan \frac{\left(\operatorname{Im} \tilde{B}_{0} \tilde{V}_{0}^{*}\right)}{\left(\operatorname{Re} \tilde{B}_{0} \tilde{V}_{0}^{*}+\alpha V_{0}^{2}+\ldots\right)}$. In practice, whatever convention is assumed for the $V$ and $B$ (which is positive or negative for approaching $V$ etc.), the crosstalk makes the two vectors more parallel (see Moretti \& Severino 2002) and the relative phase be closer to $0^{\circ}$ or $180^{\circ}$. This means that, when phases close to $90^{\circ}$ are measured, these

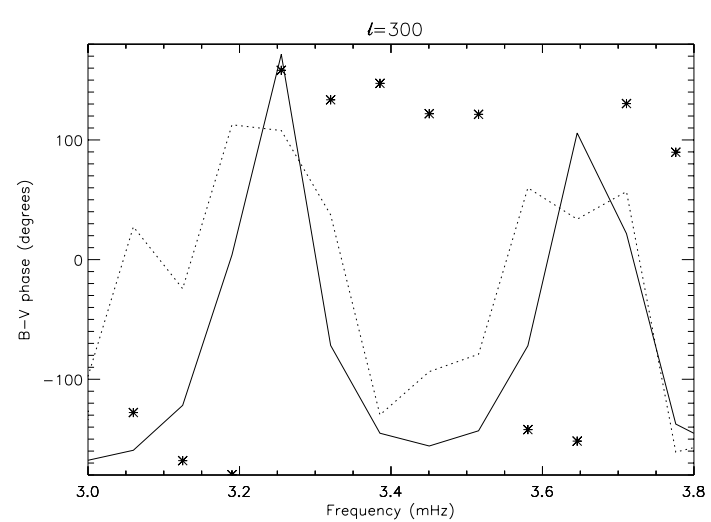

Fig. 10. The $B-V$ phase difference at $\ell=300$ in correspondence of a ridge (stars). The velocity power (solid curve, $y$-axis from 0 to $\left.1\left(\mathrm{~m} \mathrm{~s}^{-1}\right)^{2} / \mathrm{Hz}\right)$ and the coherence (dotted, $y$-axis from 0 to 0.2 ) are also shown.
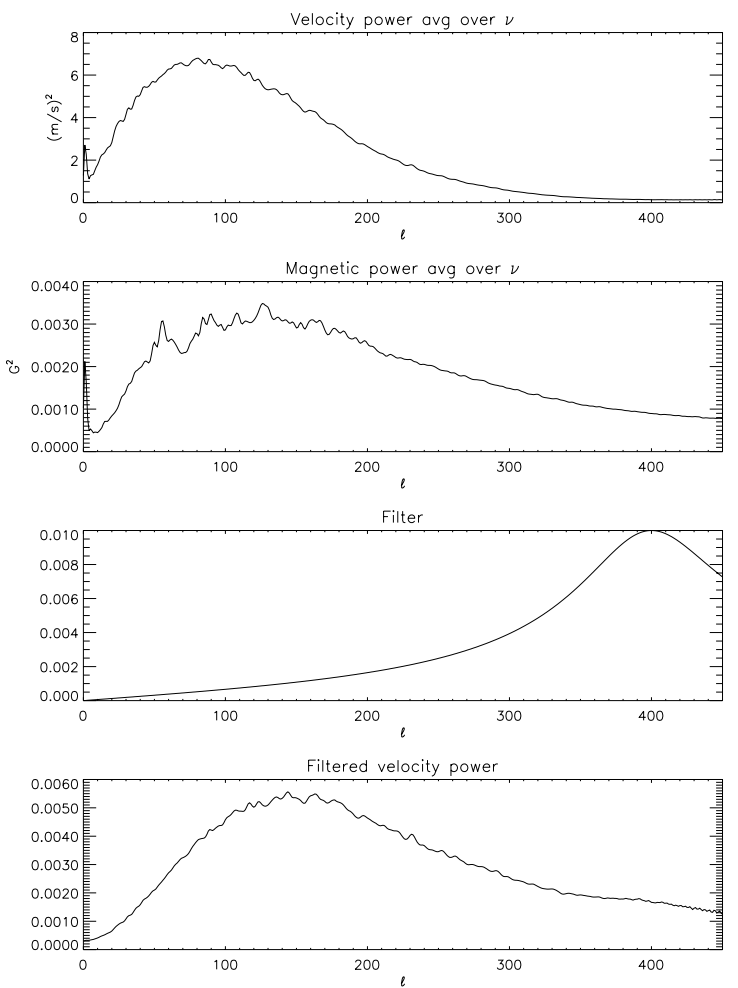

Fig. 11. From top to bottom: 1) the velocity $\ell-v$ diagram averaged over $v$. 2) The magnetic $\ell-v$ diagram averaged over $v$. 3) A $R L C$ filter with $1 / L C=\ell^{2}=160000$ and $R / L=100$. 4) The velocity power shown in the first panel as transmitted through the $R L C$ filter in the third panel (this curve should reproduce the magnetic power shown in the second panel).

values can be considered as "true". On the contrary, when $0^{\circ}$ or $180^{\circ}$ are obtained, the crosstalk term has to be estimated.

The $B-V$ phase and coherence (computed as in Oliviero et al. 1999) $\ell-v$ diagrams are shown in Fig. 8. Only 20\% of power and coherence of the ridge pattern at higher $\ell$ s can be interpreted as generated by the crosstalk terms. The $B-V$ phase difference, if averaged over the ridge area, is close to zero, but seems to show a step-like behaviour close to the power peaks (see Fig. 10). Where the coherence is low, it does not 
mean the $B$ and $V$ are not coupled but simply the spherical harmonic decomposition is not representing the resonant global behaviour for the magnetic field as well as for the velocity.

\section{Discussion}

In order to investigate the general behaviour of the power distribution at different spatial scales, the $\ell$ dependence of the magnetic power $\Delta B^{2}(\ell)$ obtained as the average over the frequencies has been performed (hereafter $\Delta B^{2}$ ). We adopt the following approach to give a possible interpretation of the results: we assume that, at the formation layer of the NaI D lines, $\Delta B^{2}$ is mainly driven by the convective and oscillatory motions. We treat the solar atmosphere as an electric circuit. We used a $C R$ and a $R L C$ to reproduce $\Delta B^{2}$ as output when $\Delta V^{2}$ is chosen as input. The CR circuit cannot explain the increase in the high- $\ell$ values as measured, even if the very low crosstalk contribution at the highest $\ell \mathrm{s}$ is taken into account. Indeed, the $R L C$ parameters can be tuned in order to match the results (Fig. 11). A $1 / L C=160000(\ell=400)$ and $R / L=100$ have been introduced to obtain an output that is most similar to the $\Delta B^{2}$. This implies the presence of a magnetic structure at spatial scales of the order of $10000 \mathrm{~km}$ to produce the right resonance filter (that is approx. 10 arcsec at disk center). The spatial resolution of the data is limited to $4 \mathrm{arcsec} / \mathrm{pix}(\ell$ max measured $=460)$ and higher resolution data with the full-disk configuration are needed to confirm this conclusion.

\section{Conclusions}

The properties of the global magnetic oscillations have been shown.

A pixel-by-pixel analysis confirms the $3 \mathrm{~min}$ power corresponding to spots and plages.

The $\ell-v$ diagram for the magnetic power shows two bumps at the three and five-minute bands. The possible crosstalk between velocity and magnetic field cannot reproduce the properties of the spectra.

A " $p$-mode"-like ridge pattern has been detected at $\ell \mathrm{s}>$ 200.

The average $\ell$ dependence of the magnetic power spectrum is preliminary interpreted as a resonant response to the velocity power at spatial scales corresponding to $\ell=400$.

Acknowledgements. P. F. M. thanks Federica Brandizzi.

We thank S. Jefferies and C. Lindsey for many fruitful discussions, M. Oliviero and the GRASP group for providing the $\ell-v$ packages. We also thank Thomas Pettauer.

\section{References}

Beckers, J. M, \& Schultz, R. B. 1972, Sol. Phys., 27, 61

Bellot Rubio, L. R., Rodriguez Hidalgo, I., Collados, M., Khomenko, E., \& Ruiz Cobo, B. 2001, AJ, 560, 1010

Bogdan, T. J., Braun, D. C., Lites, B. W., \& Thomas, J. H. 1996, AJ, 492, 379

Braun, D. C., \& Lindsey, C. 1999, AJ, 513, 79

Cacciani, A., \& Moretti, P. F. 1997, Sol. Phys., 175, 1

Cacciani, A., Di Martino, V., Jefferies, S. M., \& Moretti, P. F. 1998, ESA SP-418, 617

Cacciani, A., Moretti, P. F., Messerotti, M., et al. 1999, in Motions in the Solar Atmosphere, ed. A. Hanslmeier, \& M. Messerotti (Kluwer Academic Publishers), 239, 271

Damé, L., Gouttebroze, P., \& Malherbe, J. M. 1984, A\&A, 130, 331

Garcia, R. A., Boumier, P., Charra, J., et al. 1999, A\&A, 346, 626

Hasan, S. S., Kalkofen, W., \& van Ballegooijen, A. A. 2000, AJ, 535, L67

Horn, T., Staude, J., \& Landgraf, V. 1997, Sol. Phys., 172, 69

Jain, R., \& Haber, D. 2002, A\&A, 387, 1092

Lites, B. W., White, O. R., \& Zweibel, E. G. 1982, AJ, 253, 386

Lites, B. W. 1986, AJ, 301, 1005

Lites, B. W. 1992, in Sunspots: Theory and Observations, ed. J. H. Thomas, \& N. O. Weiss (Kluwer), 261

Lites, B. W., Thomas, J. H., Bogdan, T. J., \& Cally, P. S. 1998, AJ, 497, 464

Lites, B. W., Rutten, R. J., \& Berger, T. E. 1999, AJ, 517, 1013L

Marmolino, C., Oliviero, M., Severino, G., \& Smaldone, L. A. 1997, A\&AS, 125, 381

Moretti, P. F., \& the MOF Development Group 2000, Sol. Phys., 196, 51

Moretti, P. F., Cacciani, A., Hanslmeier, A., et al. 2001, A\&A, 372, 1038

Moretti, P. F., \& Severino, G. 2002, A\&A, 384, 638

Norton, A. A., \& Ulrich, R. K. 2000, Sol. Phys., 192, 403

Norton, A. A., Ulrich, R. K., \& Liu, Y. 2001, AJ, 561, 435

Oliviero, M., Severino, G., Straus, Th., Jefferies, S. M., \& Appourchaux, T. 1999, AJ, 516, 450

Ruedi, I., Solanki, S. K., Stenflo, J. O., Tarbell, T., \& Sherrer, P. H. 1998, A\&A, 335, L97

Scherrer, P. H., Wilcox, J. M., Svalgaard, L., et al. 1987, Sol. Phys., 55,63

Schmieder, B., Hofmann, A., \& Staude, J. 2000, Book Review: Third advances in solar physics Euroconference: magnetic fields and oscillations / Astronomical Society of the Pacific 1999, The Observatory, 120(1159), 420

Thomas, J. H., Cram, L. E., \& Nye, A. H. 1982, Nature, 297, 485

Thomas, J. H., \& Stanchfield, D. C. 2000, AJ, 537, 1086

Ulrich, R. K., Henney, C. J., Schimpf, S., et al. 1993, A\&A, 280, 268

Ulrich, R. K. 1996, AJ, 465, 436

Zhugzhda, Y. D., Balthasar, H., \& Staude, J. 2000, A\&A, 355, 347 